\title{
Autophagy inhibition enhances sensitivity of endometrial carcinoma cells to paclitaxel
}

\author{
SUILING LIU and XIAOMAO LI \\ Department of Obstetrics and Gynecology, The Third Affiliated Hospital, \\ Sun Yat-sen University, Guangzhou 510630, P.R. China
}

Received January 27, 2015; Accepted March 4, 2015

DOI: $10.3892 /$ ijo.2015.2937

\begin{abstract}
Autophagy has been shown to be involved in cancer cell resistance to chemotherapy. Paclitaxel, a widely used chemotherapeutic drug, was demonstrated to induce autophagy in various cancer cells. Therefore, we sought to evaluate the role of autophagy on the paclitaxel-induced cell death in endometrial carcinoma. In this study, we found that paclitaxel induced autophagy in paclitaxel-insensitive HEC-1A and JEC cells, exhibiting an increased microtubule associated protein 1 light chain 3 (LC3)-II/LC3-I ratio, a decrease in p62/ SQSTM1 abundance, the upregulation of Beclin 1 expression and punctate dots of yellow fluorescent protein (YFP)-LC3 in the cytosol. Paclitaxel-mediated cell death was further potentiated by pretreatment with autophagy inhibitor chloroquine (CQ) or shRNA against the autophagic gene beclin 1. Moreover, paclitaxel stimulated reactive oxygen species (ROS) generation, and inhibition of the ROS by antioxidant $\mathrm{N}$-acetyl-cysteine (NAC) blocked paclitaxel-induced autophagy, indicating that paclitaxel-induced autophagy in endometrial carcinoma cells is mediated by ROS. These findings suggest that paclitaxelelicited autophagic response plays a protective role that impedes the eventual death of endometrial carcinoma cell, and that autophagy-inhibitor therapy could be an effective and potent strategy to improve paclitaxel treatment outcomes in the treatment of endometrial carcinoma.
\end{abstract}

\section{Introduction}

Endometrial carcinoma is the most common malignancy of the female reproductive tract. Although the majority of

Correspondence to: Dr Xiaomao Li, Department of Obstetrics and Gynecology, The Third Affiliated Hospital, Sun Yat-sen University, 600 Tianhe Rd, Guangzhou 510630, P.R. China

E-mail: tigerlee777@163.com

Abbreviations: LC3, microtubule associated protein 1 light chain 3; YFP, yellow fluorescent protein; $\mathrm{CQ}$, chloroquine; ROS, reactive oxygen species; NAC, N-acetyl-L-cysteine

Key words: paclitaxel, autophagy, chloroquine, ROS, endometrial carcinoma patients with early-stage disease are cured with surgery and/or radiation therapy, advanced endometrial carcinoma continues to portend a poor prognosis. Chemotherapy is an important treatment option for women with advanced and recurrent endometrial cancer or women in early stage with high-risk factor. Enhancing the sensitivity of endometrial carcinoma cells to chemotherapy agents could lead to an improved clinical outcome.

The combination of paclitaxel and carboplatin is currently regarded as the standard regimen of endometrial carcinoma based on its favourable toxicity profile and good response rates (1-4). Paclitaxel is a potent mitotic inhibitor and inducer of apoptosis. Paclitaxel binds to $\beta$-tubulin thereby inhibiting microtubule depolymerization, which ultimately results in cell cycle arrest in G2/M phase and subsequent apoptosis. Furthermore, previous studies have shown that paclitaxel can induce autophagy in many cancer cell lines. Autophagy is a tightly regulated process in which a cell engulfs cytoplasmic constituents within a double-membrane vacuole known as an autophagosome, and delivers them to the lysosome for degradation. Degradation of the contents within the autophagosome generates free amino and fatty acids that can be recycled intrinsically or delivered systemically to distant sites within the organism. Therefore, the process of autophagy promotes cellular homeostasis and viability by way of maintaining quality control of both proteins and organelles.

Early studies demonstrate that a variety of chemical insults can activate autophagy in vitro as well as in vivo in certain types of cancers (5). Autophagy induced by certain cancer therapeutics may cause the undesired effect of protecting cancer cells from apoptosis $(6,7)$. As increasing evidence suggests that autophagy promotes cancer cell survival after chemotherapy and that the inhibition of autophagy resensitizes resistant cancer cells to anticancer agents, it has been proposed that suppression of the autophagic pathway may be combined with conventional or experimental antitumor regimens to achieve increased efficacy, thereby allowing for lower dosage and limited side effects. Conversely, some cytotoxic drugs have been discovered to trigger progressive autophagy that ultimately leads to autophagic cell death, particularly in apoptosis-defective cells. As such, induction of autophagic cell death through over-stimulation of autophagy has also been considered a potential therapeutic strategy to eradicate cancer cells. 
Several studies have demonstrated that the pro-apoptotic drug paclitaxel can elicit an autophagic response that actually plays a protective role, ultimately impeding cell death (8-10). Upregulation of the autophagic pathway has also been shown to be associated with paclitaxel resistance in breast cancer cells (11). However, Eum and Lee (12) found that both autophagy and apoptosis act as cooperative partners to induce cell death in paclitaxel treated v-Ha-ras-transformed NIH3T3 cells. Veldhoen et al (13) reported that in non-mitotic paclitaxel-treated MCF-7 cells, autophagosomes were generated to sensitize cells to paclitaxel toxicity. Nonetheless, paclitaxel-induced autophagy and its effect on paclitaxelassociated cytotoxity in endometrial carcinoma cells have not been described. In this study, we focused on the effects of autophagy on paclitaxel-insensitive endometrial carcinoma cell lines. We found that paclitaxel induced reactive oxygen species (ROS)-mediated cytoprotective autophagy in endometrial carcinoma cells. Furthermore, we demonstrated that the suppression of autophagy using chloroquine (CQ) increased paclitaxel-induced cell death, thereby suggesting a new approach for enhancing the chemotherapeutic efficacy of paclitaxel in endometrial carcinoma.

\section{Materials and methods}

Cell culture. HEC-1A human endometrial carcinoma cells were purchased from the American Type Culture Collection (Manassas, VA, USA), AN3CA human endometrial carcinoma cells from the Shanghai Cell Collection, Chinese Academy of Sciences (Shanghai, China) and JEC human endometrial carcinoma cells from the China Center for Type Culture Collection (Wuhan, China). Ishikawa human endometrial carcinoma cells were kindly provided by Professor Zehua Wang (Department of Obstetrics and Gynecology, Union Hospital, Tongji Medical College, Huazhong University of Science and Technology, Wuhan, China). Cells were cultured in Dulbecco's modified Eagle's medium (Gibco, Carlsbad, CA, USA) supplemented with $10 \%$ fetal bovine serum (Hyclone, South Logan, UT, USA) with varying concentrations of paclitaxel (BristolMyers Squibb, New York, NY, USA), and/or chloroquine (CQ) (Sigma-Aldrich, St. Louis, MO, USA), and/or N-acetylL-cysteine (NAC) (Sigma-Aldrich) at $37^{\circ} \mathrm{C}$ in a humidified chamber with $5 \%$ carbon dioxide.

Cell viability assay. Cell viability were measured using a 3-(4,5-dimethylthiazol-2-yl)-2,5-diphenyltetrazolium bromide (MTT) assay (MP, CA, USA). Cells of different cell lines were seeded in 96-well plates at varied density $\left(4 \times 10^{3}-1.1 \times 10^{4}\right.$ per well) and grown to $70 \%$ confluence. Paclitaxel was added to culture medium at the indicated concentrations. After treatment, cells were cultured for an additional $24 \mathrm{~h}$. Twenty microliters of MTT $(5 \mathrm{mg} / \mathrm{ml})$ was added to each well, and cells were subsequently incubated at $37^{\circ} \mathrm{C}$ for an additional $4 \mathrm{~h}$. Crystals were dissolved in $150 \mu \mathrm{l}$ of DMSO. Absorbance was measured at a wavelength of $490 \mathrm{~nm}$ using a microplate reader (Bio-Tek, ELx800, USA).

Confocal microscopy. Cells were grown on glass coverslips and transfected with YFP-LC3. Twenty-four hours after transfection, cells were treated with paclitaxel and analyzed after an additional $24 \mathrm{~h}$. Cells were fixed with $4 \%$ paraformaldehyde in PBS for $30 \mathrm{~min}$ at room temperature, then mounted on slides in anti-fading solution and stored at $4^{\circ} \mathrm{C}$. Slides were subsequently examined under a laser-scanning confocal microscope (Olympus, FV-1000, Japan).

Immunoblot analysis. Cells were seeded in 6-well plates and grown to $70 \%$ confluence. Cells were harvested $24 \mathrm{~h}$ after the indicated treatment. Lysates were prepared by dissolving cell pellets in $100 \mu 1$ lysis buffer [ $20 \mathrm{mM} \mathrm{Na} \mathrm{PO}_{4}$ (pH 7.4), $150 \mathrm{mM} \mathrm{NaCl}, 1 \%$ Triton $\mathrm{X}-100,1 \%$ aprotinin, $1 \mathrm{mM}$ phenymethysulfonyl fluoride, $10 \mathrm{mg} / \mathrm{ml}$ leupeptin, $100 \mathrm{mM}$ $\mathrm{NaF}$ and $\left.2 \mathrm{mM} \mathrm{Na}_{3} \mathrm{VO}_{4}\right]$. Cell lysates were centrifuged for $10 \mathrm{~min}$ at $14,000 \mathrm{~g}$. Protein concentrations in the supernatant were determined by BCA protein assay. Total protein $(25 \mu \mathrm{g})$ was separated by SDS-PAGE, then transferred to PVDF membranes. Membranes were blocked with non-fat dry milk (5\%) in TBST (10 mM Tris-HCl, pH 7.4, $150 \mathrm{mM}$ $\mathrm{NaCl}, 0.1 \%$ Tween-20), and incubated with primary antibodies for $1 \mathrm{~h}$ at $4^{\circ} \mathrm{C}$. Primary antibodies included those to detect microtubule associated protein 1 light chain 3 (LC3) (Novus Biologicals Inc., Littleton, CO, USA), P62/SQSTM1 (Santa Cruz Biotechnlogy, Inc., Santa Cruz, CA, USA) and Beclin 1 (Cell Signaling, Beverly, MA, USA). After primary antibody incubation, membranes were washed three times with TBST for $30 \mathrm{~min}$, then incubated with secondary antibody conjugated with horseradish peroxidase for $2 \mathrm{~h}$ at room temperature. After washing out the secondary antibody, the bound antibody complex was detected using an ECL chemiluminescence reagent.

RNA interference. Protein depletion through RNA-mediated interference (RNAi) was mediated using the GV246 shRNA system. The target sequences for beclin 1-specific shRNA were 5'-UGGAAUGGAAUGAGAUUAATT-3' and 5'-GCU CAGUAUCAGAGAGAAUTT-3' (GeneBank accession no. NM003766.2). Lentiviruses were generated by co-transfection of GV246-shRNA plasmids with lentivirus plasmids PIK into 293 T cells by lipidosome. Lentiviruses were collected in high-serum media at 72 and $96 \mathrm{~h}$ following transfection. HEC-1A cells were transduced with lentiviruses and $8 \mu \mathrm{g} / \mathrm{ml}$ polybrene (hexadimethrine bromide, Sigma-Aldrich) followed by incubation with virus at $37^{\circ} \mathrm{C}$ for $4-6 \mathrm{~h}$. shRNA-transduced cells were selected with $1 \mu \mathrm{g} / \mathrm{ml}$ puromycin for $72 \mathrm{~h}$. Knockdown was assessed by western blotting at an appropriate time-point each time the experiment was replicated.

Measurement of intracellular ROS. Reactive Oxygen Species Assay kit was purchased from Beyotime (Beijing, China). Intracellular ROS levels were measured by detecting the conversion of cell permeable 2, 7-dichlorofluorescein diacetate (DCFH-DA) to fluorescent dichlorofluorescein (DCF). Cells were seeded in 12-well plates and treated with the experimental reagents indicated for $24 \mathrm{~h}$. After washing several times with PBS, cells were incubated with $5 \mu \mathrm{M} \mathrm{2}$ ', 7'-dichlorofluorescein diacetate (DCFH-DA) for $1 \mathrm{~h}$ at $37^{\circ} \mathrm{C}$. After a second wash step with PBS, positively stained cells were analyzed at an excitation wavelength of $488 \mathrm{~nm}$ and an emission wavelength of $525 \mathrm{~nm}$ by BD FACScan flow cytometry (BD LSRII, USA). 

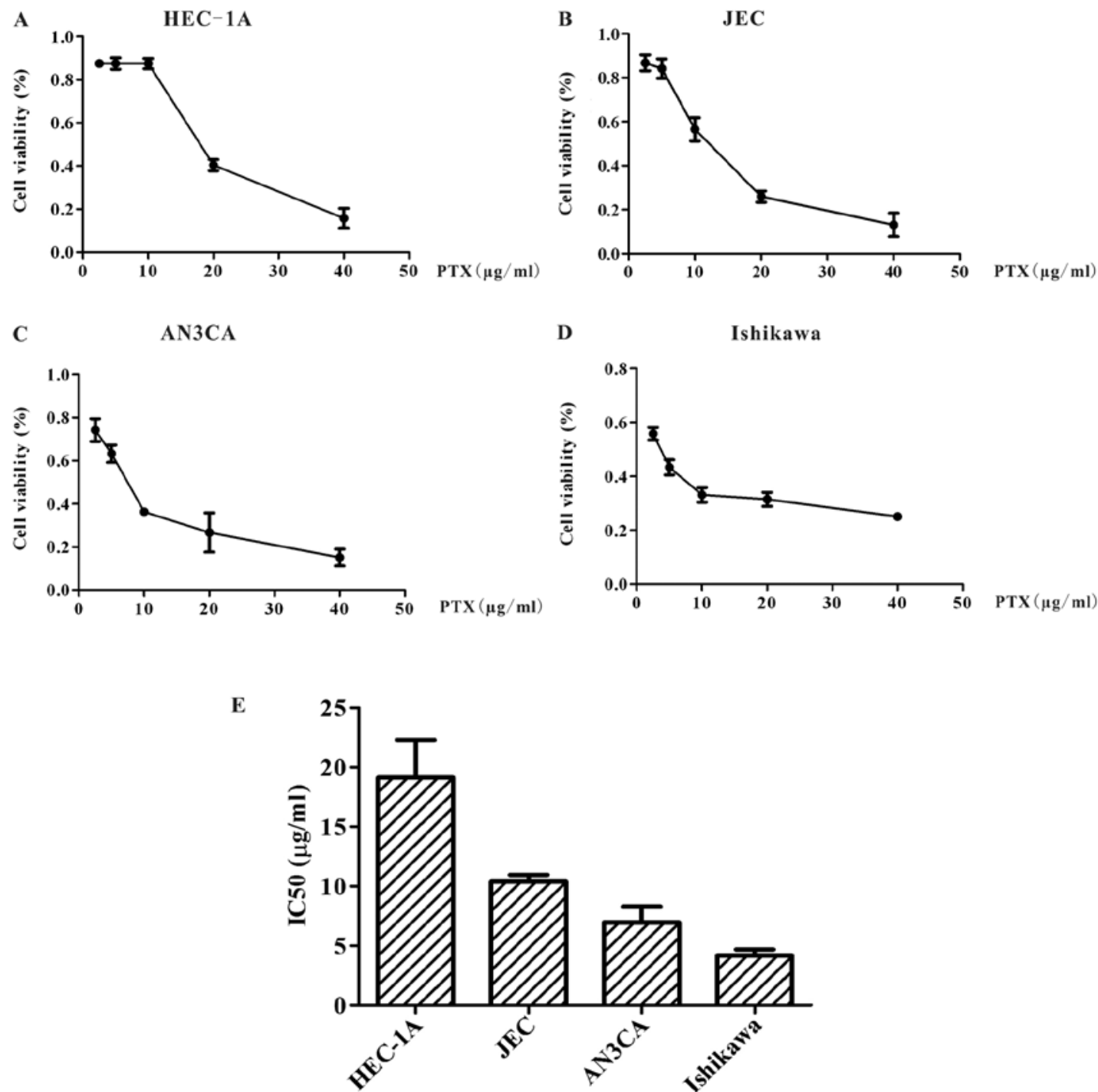

Figure 1. Cytotoxicity of paclitaxel in endometrial carcinoma cells. (A) HEC-1A, (B) JEC, (C) AN3CA, (D) Ishikawa cells were treated with various concentrations of paclitaxel for $24 \mathrm{~h}$. Cell viability was subsequently quantified using the MTT assay. (E) $\mathrm{IC}_{50}$ values were determined for each cell line. PTX, paclitaxel.

\section{Results}

Paclitaxel induces autophagy in insensitive endometrial carcinoma cells. Four endometrial carcinoma cell lines including HEC-1A, JEC, AN3CA and Ishikawa cells were chosen to investigate paclitaxel resistance in endometrial carcinoma. MTT assay was used to detect the $\mathrm{IC}_{50}$ values of paclitaxel on each endometrial carcinoma cell line. As shown in Fig. 1, upon exposure to paclitaxel for $24 \mathrm{~h}$, the $\mathrm{IC}_{50}$ of HEC-1A, JEC, AN3CA and Ishikawa cells was $19.14 \pm 3.16 \mu \mathrm{g} / \mathrm{ml}$ (Fig. 1A), $10.39 \pm 0.55 \mu \mathrm{g} / \mathrm{ml}$ (Fig. 1B), 6.95 $\pm 1.33 \mu \mathrm{g} / \mathrm{ml}$ (Fig. 1C) and $4.15 \pm 0.52 \mu \mathrm{g} / \mathrm{ml}$ (Fig. 1D), respectively. HEC-1A proved to be the least sensitive to paclitaxel as compared to other cell lines, due to its relatively high $\mathrm{IC}_{50}$ value (Fig. 1E). Therefore, we chose HEC-1A as the paclitaxel-resistant endometrial carcinoma cell line for subsequent studies. We sought to determine whether paclitaxel could induce autophagy in HEC-1A cells. LC3 and P62 were used to detect autophagy. LC3 is an important autophagy marker recruited to the autophagosomal membrane. LC3 has two isoforms, LC3-I and LC3-II. During autophagy, LC3-I is conjugated to autophagic membrane-associated phosphatidylethanolamine and converted to LC3-II. Since LC3-II is localized in autophagosomal membranes throughout the autophagy process, LC3-II expression is regarded as a good marker for autophagosome formation. Increased LC3-II expression, especially an increased LC3-II/ LC3-I ratio, may indicate the occurrence of autophagy $(14,15)$. P62/SQSTM1 is a highly conserved scaffolding protein involved in the transportation of ubiquitinylated proteins destined for proteosomal degradation $(15,16)$. A decrease in p62 expression could indicate an early autophagic degradation, which makes it an alternative method for detecting autophagic flux (17). Using immunoblot analysis, we observed that paclitaxel induced a significant conversion of LC3I to LC3II, as well as an alteration of P62 expression in HEC-1A cells. As shown in Fig. 2A and B, in cells treated with paclitaxel, the expression of LC3-II increased in a dose- and time-dependent manner, and the expression of P62 dramatically decreased, as compared to control cells. These results demonstrate that paclitaxel induces autophagy in HEC-1A cells. 
A

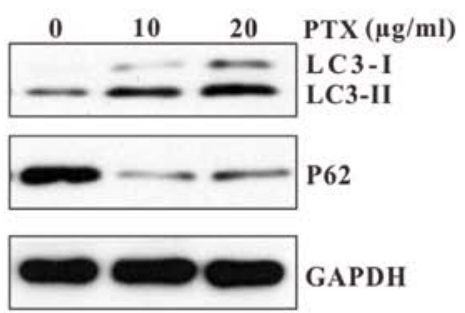

$\begin{array}{llllll}\text { B } & 0 & 6 & 12 & 24 & \text { Time (h) }\end{array}$

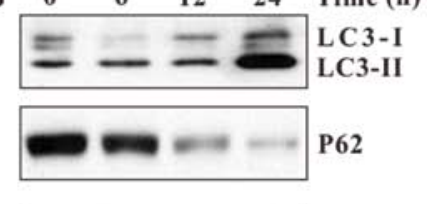

50 GAPDH

C

HEC-1A
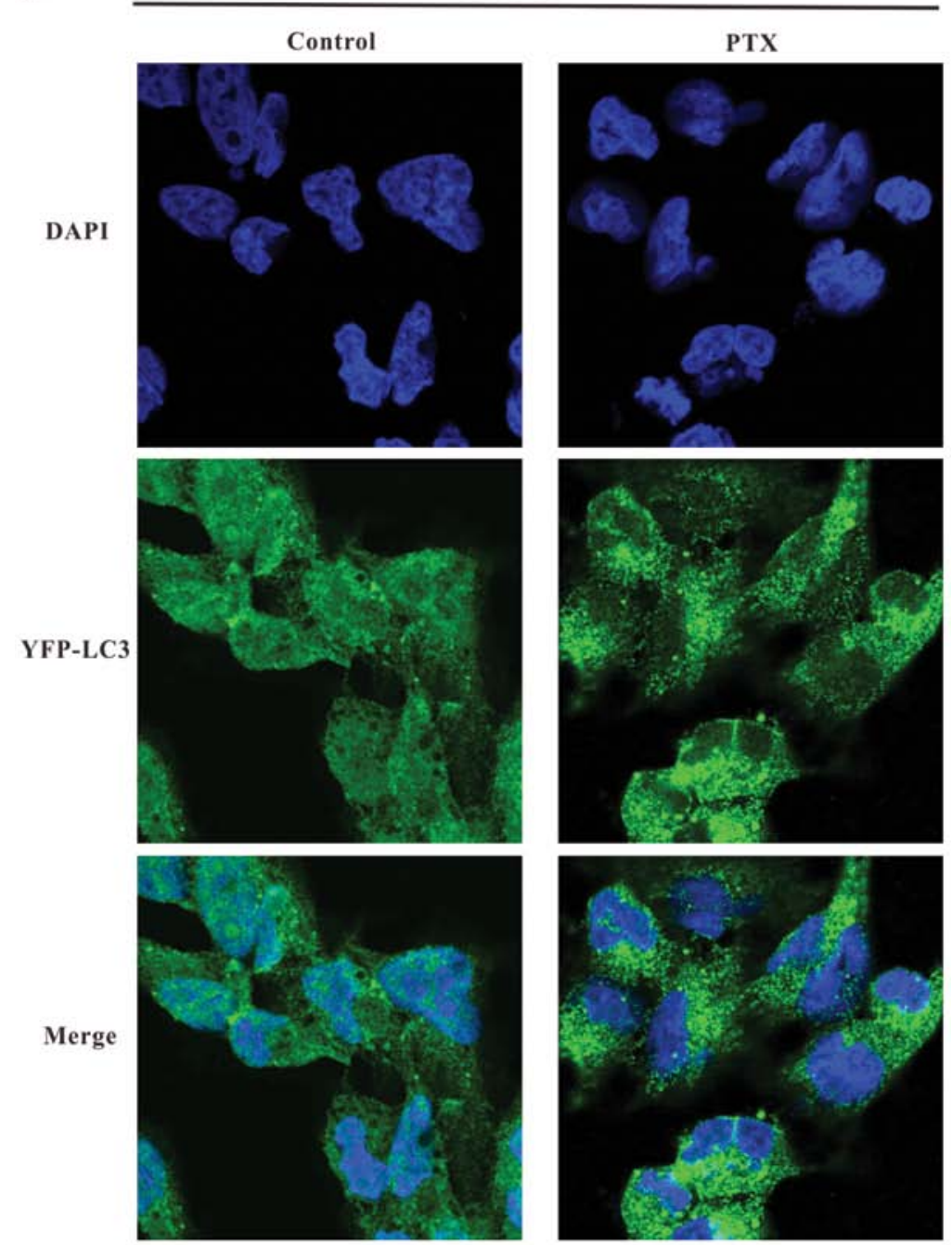

Figure 2. Paclitaxel induces autophagy in HEC-1A cells. (A) HEC-1A cells were treated with 10 or $20 \mu \mathrm{g} / \mathrm{ml}$ paclitaxel for $24 \mathrm{~h}$. Expression levels of LC3 and P62 protein were analyzed by immunoblotting. GAPDH was used as internal control. (B) HEC-1A cells were treated with $20 \mu \mathrm{g} / \mathrm{ml}$ paclitaxel for the indicated intervals. Expression levels of LC3 and P62 protein were analyzed by immunoblotting. GAPDH was used as internal control. (C) HEC-1A cells were treated with $20 \mu \mathrm{g} / \mathrm{ml}$ paclitaxel or vehicle after transfection with YFP-LC3 for $24 \mathrm{~h}$. YFP-LC3 expression and localization were analyzed using confocal microscopy. Nuclei were detected with DAPI.

To confirm the induction of autophagy in HEC-1A cells, we examined the formation of autophagosomes after paclitaxel treatment using YFP-LC3, an LC3 expression construct fused to a yellow fluorescent protein. HEC-1A cells were transfected with YFP-LC3 and exposed to paclitaxel. As shown in Fig. 2C, in control cells, YFP -LC3 was evenly distributed throughout the entire cytoplasm. However, in paclitaxel treated cells, the punctate dots of YFP-LC3 were detectable in the cytosol, indicating the association of YFP-LC3 with autophagosomal membranes, which suggests the induction of autophagy.
We extended our studies to test whether autophagy was induced in the other endometrial carcinoma cell lines. As shown in Fig. 3, the paclitaxel-induced increase in LC3-II and decrease in P62 were also exhibited in JEC cells (Fig. 3A and B), but not in AN3CA or Ishikawa cells (Fig. 3D). YFP-LC3 punctate dots were also detectable in the cytoplasm of paclitaxel-treated JEC cells (Fig. 3C). Because autophagy was only induced in HEC-1A and JEC cells which exhibited high $\mathrm{IC}_{50}$ values, we inferred that autophagy may be involved in the resistance of endometrial carcinoma cells to paclitaxel. 

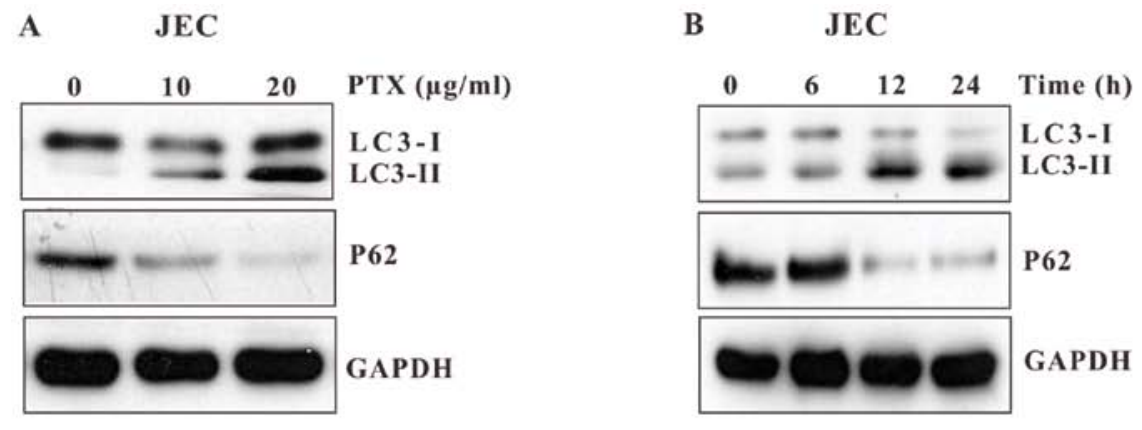

C

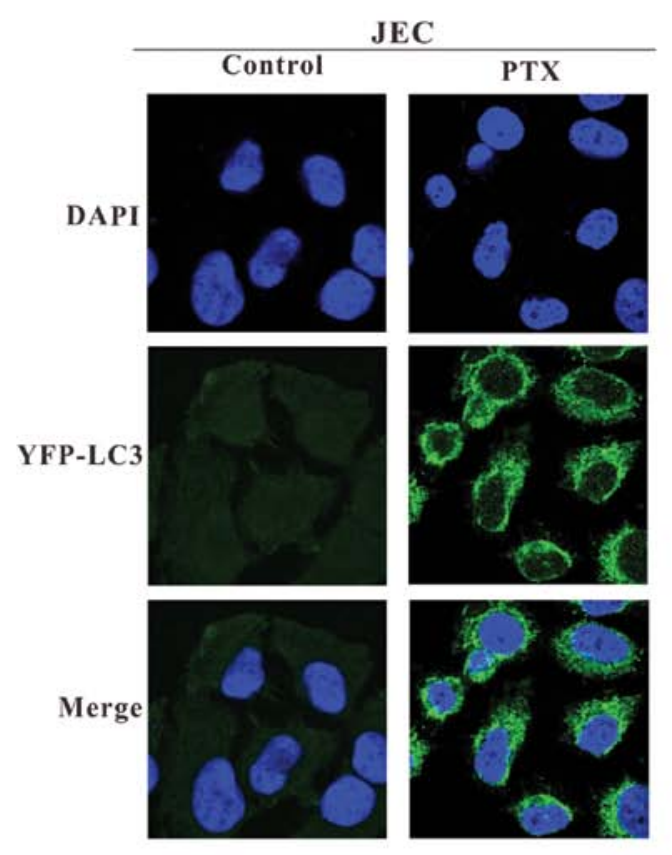

D

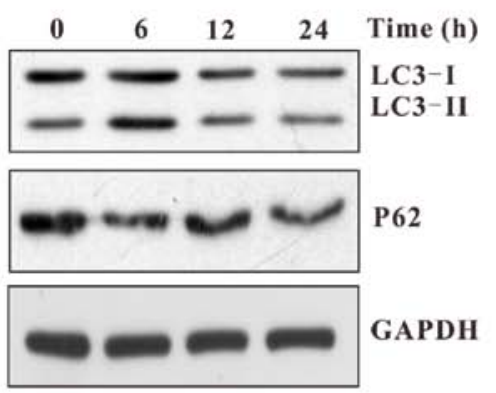

Ishikawa

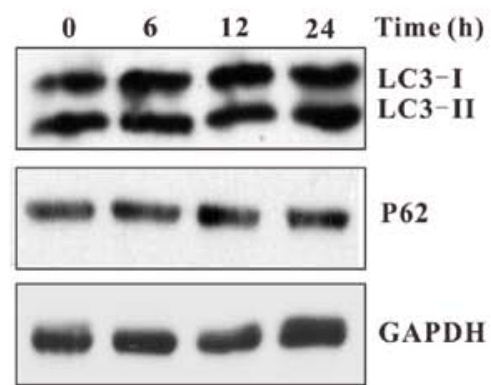

Figure 3. Paclitaxel induces autophagy in JEC cells, but not in AN3CA and Ishikawa cells. (A) JEC cells were treated with $10 \mathrm{or} 20 \mu \mathrm{g} / \mathrm{ml}$ paclitaxel for $24 \mathrm{~h}$. Expression levels of LC3 and P62 protein were analyzed by immunoblotting. GAPDH was used as internal control. (B) JEC cells were treated with $20 \mu \mathrm{g} / \mathrm{ml}$ paclitaxel for the indicated intervals. Expression levels of LC3 and P62 protein were analyzed by immunoblotting. GAPDH was used as internal control. (C) JEC cells were treated with $20 \mu \mathrm{g} / \mathrm{ml}$ paclitaxel or vehicle after transfection with YFP-LC3 for $24 \mathrm{~h}$. YFP-LC3 expression and localization were analyzed using confocal microscopy. Nuclei were detected with DAPI. (D) AN3CA and Ishikawa cells were treated with $20 \mu \mathrm{g} / \mathrm{ml}$ paclitaxel for the indicated intervals. GAPDH was used as internal control.

Knockdown of Beclin 1 by shRNA increases the cytotoxic sensitivity of endometrial carcinoma cells to paclitaxel. It has been shown that Beclin 1 and its binding partner class III phosphoinositide 3-kinase (PI3K), also named Vps34, are required for the initiation of the formation of the autophagosome. As such, we detected the expression of Beclin 1 in HEC-1A and JEC cells. We found that Beclin 1 expression increased in a time- and dose-dependent manner similarly to LC 3 and P62 in these two cell lines (Fig. 4A and B). The result indicates that paclitaxel-induced autophagy in endometrial carcinoma cells may be regulated by Beclin 1 complex.

To exploit whether Beclin 1 plays an intrinsic role in autophagy induced by paclitaxel, the effect of Beclin 1 knockdown on paclitaxel-mediated cell death was investigated. We 
A
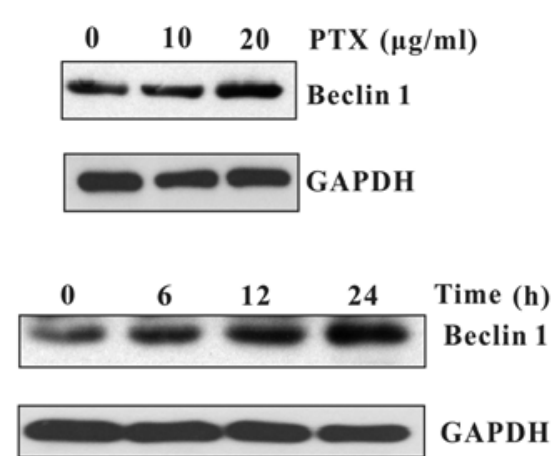

C

$$
\text { HEC-1A }
$$

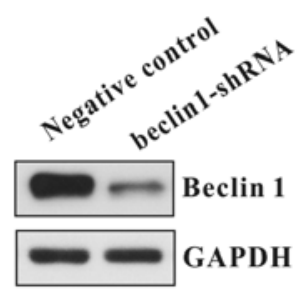

B

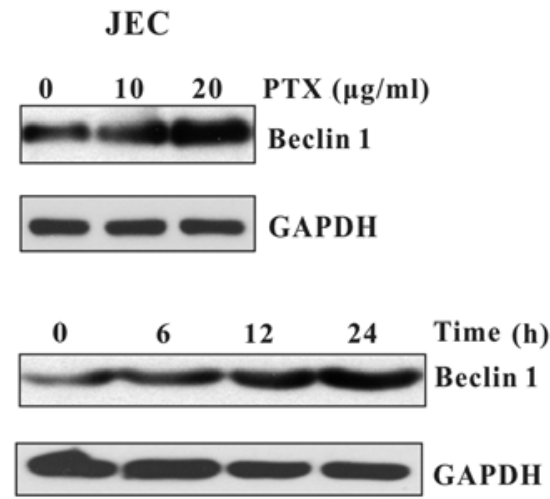

D

HEC-1A Negative control beclin 1-shRNA

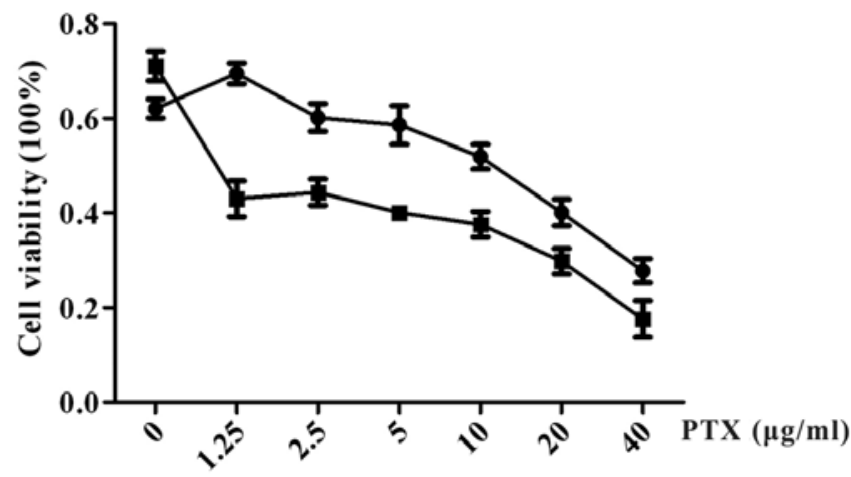

Figure 4. Paclitaxel induces Beclin 1 expression in HEC-1A and JEC cells. (A and B) Paclitaxel dose- and time-dependently induced the expression of Beclin 1. HEC-1A and JEC cells were treated with paclitaxel in the indicated concentrations for $24 \mathrm{~h}$ or treated with $20 \mu \mathrm{g} / \mathrm{ml}$ paclitaxel for the indicated times. Lysates were analyzed by immunoblotting with Beclin 1 antibody. GAPDH was used as internal control. (C) Western blot analysis of endogenous Beclin 1 in extracts from HEC-1A cells transfected with non-targeting control shRNA or Beclin 1-specific shRNA. (D) MTT assay of HEC-1A cells transfected with shRNA as above.

used shRNA to knock down Beclin 1 expression in HEC-1A cells (Fig. 4C). As shown in Fig. 4D, when Beclin 1 expression was markedly suppressed by beclin 1 shRNA, HEC-1A cells were more sensitive to paclitaxel. These data suggest that Beclin 1 is upregulated to initiate cytoprotective autophagy in endometrial carcinoma cells following paclitaxel treatment.

Inhibition of autophagy increases paclitaxel-induced cell death. To investigate the impact of autophagy on paclitaxelmediated cell death, chloroquine (CQ), an autophagy inhibitor, was used in combination with paclitaxel in endometrial carcinoma cell lines. CQ is a weak base that can be trapped in acidic vesicles, elevate intralysosomal $\mathrm{pH}$ (18), and thus block autophagosome degradation (19,20). As depicted in Fig. 5A, in immunoblot analysis, paclitaxel increased the production of LC3-II in HEC-1A and JEC cells, which was in accordance with the aforementioned results. Compared to paclitaxel treatment alone, co-treatment of paclitaxel and CQ led to accumulation of LC3-II and impaired p62 degradation. These results suggest that autophagic flux induced by paclitaxel could be inhibited by CQ in the autophagic degradation stage.

We then examined the impact of autophagy inhibition on the survival of HEC-1A and JEC cells exposed to paclitaxel.
MTT assay showed that the cell viability after exposure of HEC-1A and JEC cells to paclitaxel for $24 \mathrm{~h}$ was significantly decreased in the presence of CQ. (Fig. 5B). We also observed that in the paclitaxel + CQ group, HEC-1A cells appeared to exhibit severe shrinkage, and the number of cells was further reduced compared to the paclitaxel alone treatment group (Fig. 5C).

These results confirm that autophagy is induced to counteract the cytotoxity of paclitaxel in HEC-1A cells and JEC cells, and that the inhibition of autophagy enhances sensitivity to paclitaxel.

Paclitaxel treatment generates reactive oxygen species. As paclitaxel can trigger the generation of intracellular ROS in many cancer cell lines (21-23), we next investigated whether ROS was elevated in HEC-1A and JEC cells following paclitaxel exposure. Since DCFH-DA was often used to assess paclitaxel-induced ROS in previous studies $(21,23)$, we used DCFH-DA to measure the intracellular levels of ROS in our study. DCFH-DA can diffuse into cells through the cell membrane, and subsequently become hydrolyzed to non-fluorescent DCFH. ROS leads to oxidation of DCFH to DCF, which resulting in the emission of a fluorescent signal. 
A

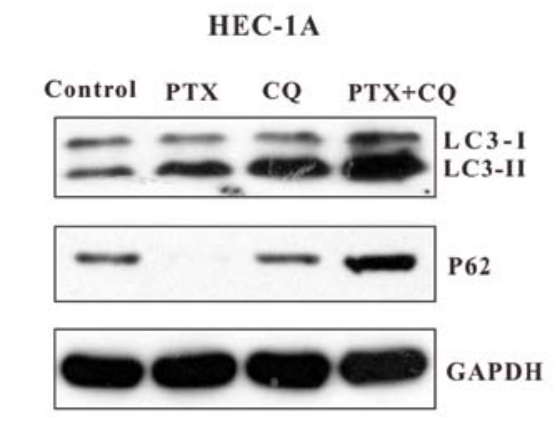

B

HEC-1A

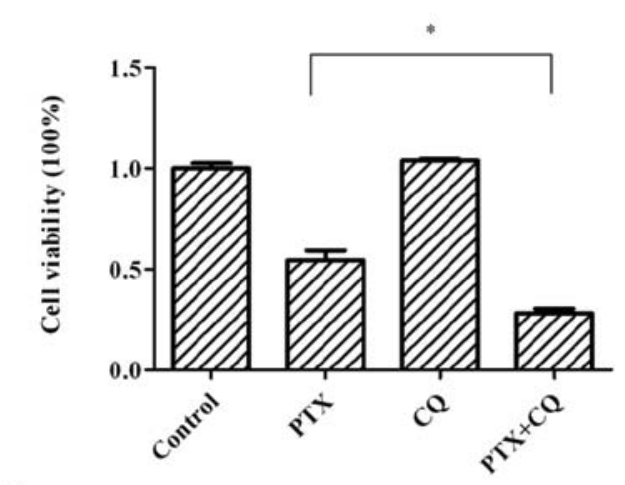

C

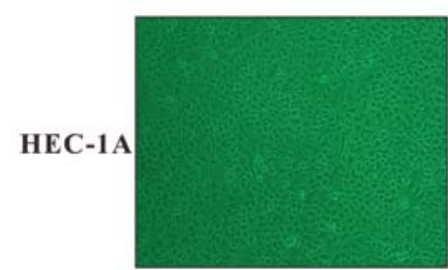

Control

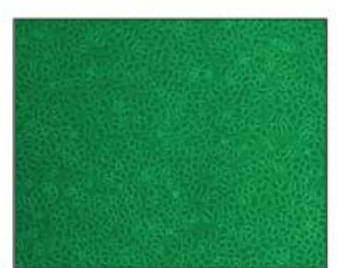

CQ

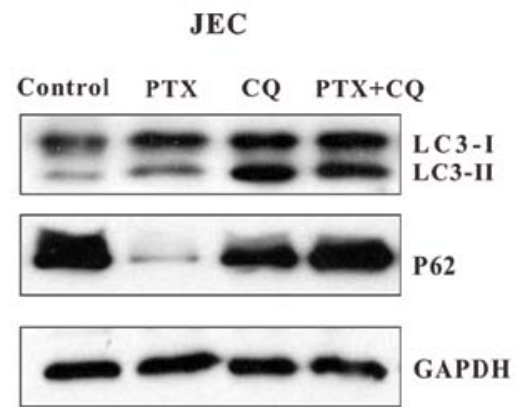

JEC
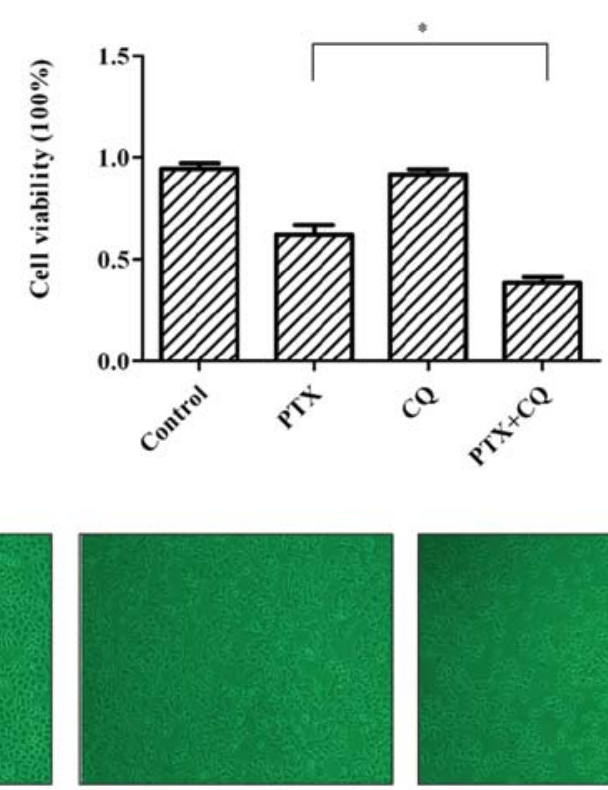

PTX

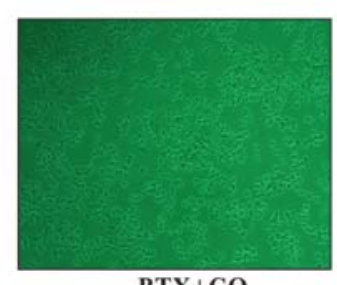

PTX+CQ

Figure 5. Increase in paclitaxel-induced cell death by inhibition of autophagy in HEC-1A and JEC cells. HEC-1A and JEC cells were pre-treated with CQ $(40 \mu \mathrm{M})$ or vehicle for $1 \mathrm{~h}$ then treated with $20 \mu \mathrm{g} / \mathrm{ml}$ paclitaxel or vehicle for $24 \mathrm{~h}$. (A) Western blot analysis of LC3 and P62 expression. (B) Cell viability evaluated using MTT assay. Data are expressed as mean \pm SD from three independent experiments. * $\mathrm{P}<0.05$. (C) Light microscopy of cellular morphology of HEC-1A cells. Original magnification, $\mathrm{x} 100$.

Our study showed that treatment with paclitaxel significantly increased ROS level (indicated by increased green fluorescence) in HEC-1A and JEC cells. The increase of ROS level was time- and dose-dependent (Fig. 6A and B). We then used NAC, a ROS scavenger, to treat HEC-1A and JEC cells together with paclitaxel. Co-treatment of NAC and paclitaxel led to attenuation of ROS level (Fig. 6C). All the results indicated that paclitaxel promotes ROS generation in HEC-1A and JEC cells.

Paclitaxel induces ROS-mediated autophagy. To examine the role of ROS in paclitaxel-induced autophagy, HEC-1A and JEC cells were co-treated with paclitaxel and NAC. As shown in Fig. 7, when co-treated with NAC, paclitaxel-induced LC3 conversion was decreased in HEC-1A and JEC cells. P62 level was elevated. This data demonstrated that when ROS was decreased, autophagy flux was blocked in the early stage. The result indicates that paclitaxel-induced autophagy in endometrial carcinoma cells is mediated by ROS.

\section{Discussion}

In the present study, we found that paclitaxel induced the generation of reactive oxygen species (ROS), which resulted in cytoprotective autophagy in insensitive endometrial carcinoma cells. Notably, we demonstrated that the suppression of autophagy using chloroquine (CQ) increased paclitaxelinduced cell death, thereby suggesting a novel approach for enhancing the chemotherapeutic effect of paclitaxel in the treatment of endometrial carcinoma.

Autophagy has been shown to be involved in mediating the resistance of cancer cells to anticancer therapy. In our study, autophagy-related properties including increased LC3-II/ LC3-I ratio, decrease in p62 abundance, and punctate dots of YFP-LC3 in cytosol, were obvious in paclitaxel-insensitive endometrial carcinoma cells HEC-1A and JEC but not in the two paclitaxel-responsive endometrial carcinoma cell lines, AN3Ca and Ishikawa. Subsequent experiments demonstrated that inhibiting autophagy resulted in an increase in paclitaxel- 
A

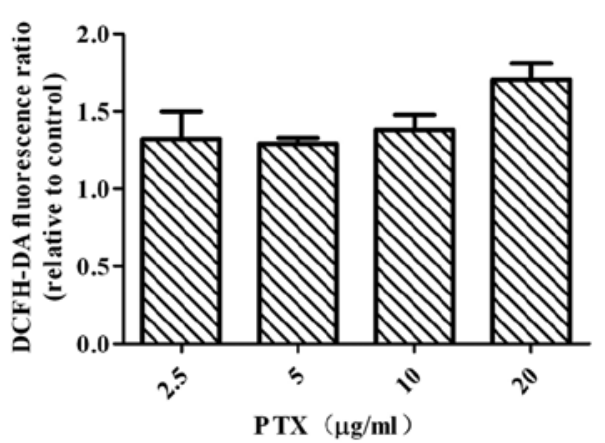

B

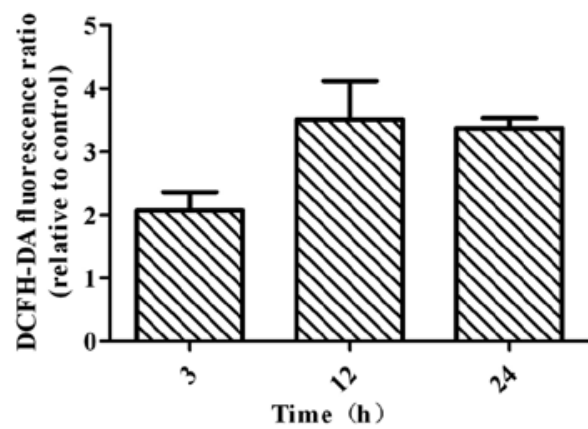

C

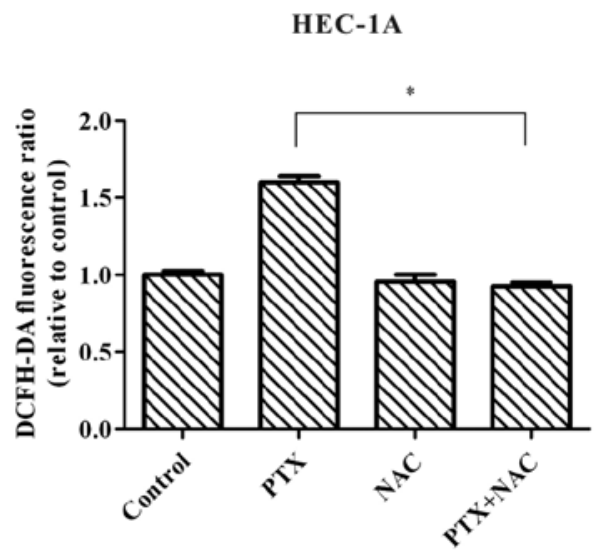

JEC

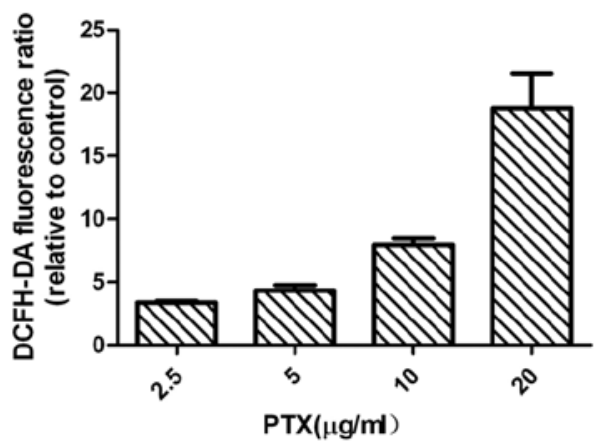

JEC

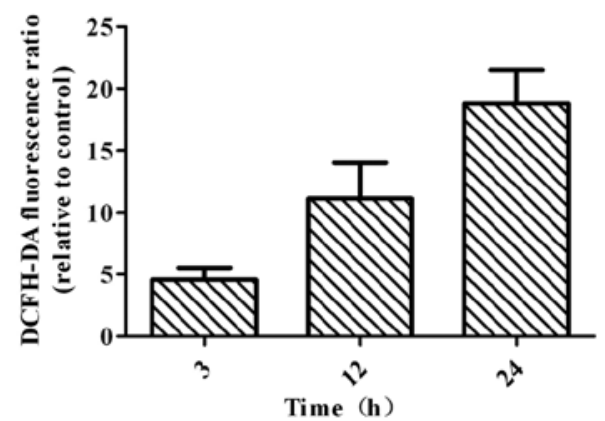

JEC

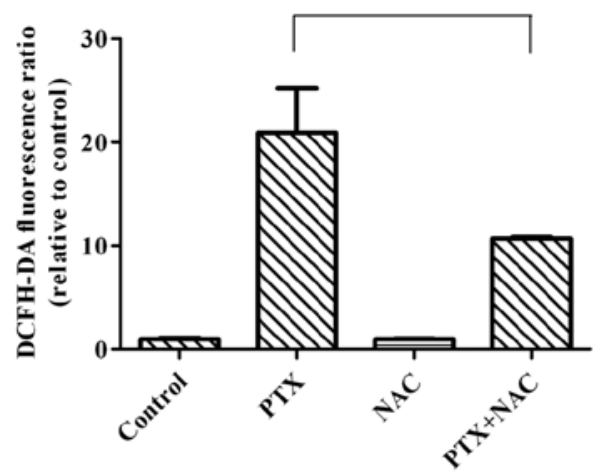

Figure 6. Effects of paclitaxel on intracellular ROS production in HEC-1A and JEC cells. ROS levels were measured by flow cytometry following DCFH-DA treatment. (A) HEC-1A and JEC cells were treated with various concentrations of paclitaxel for $24 \mathrm{~h}$. (B) HEC-1A and JEC cells were treated with $20 \mu \mathrm{g} / \mathrm{ml}$ paclitaxel for the indicated intervals. (C) HEC-1A and JEC cells were pre-incubated with NAC $(1 \mathrm{mM})$ or vehicle for $1 \mathrm{~h}$ before treatment with paclitaxel $(20 \mu \mathrm{g} / \mathrm{ml})$ for $24 \mathrm{~h}$. Results are expressed as mean $\pm \mathrm{SD}$ of 3 independent experiments. ${ }^{*} \mathrm{P}<0.05$.

HEC-1A

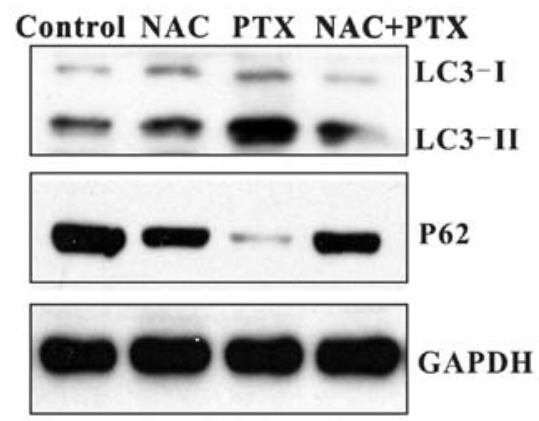

JEC

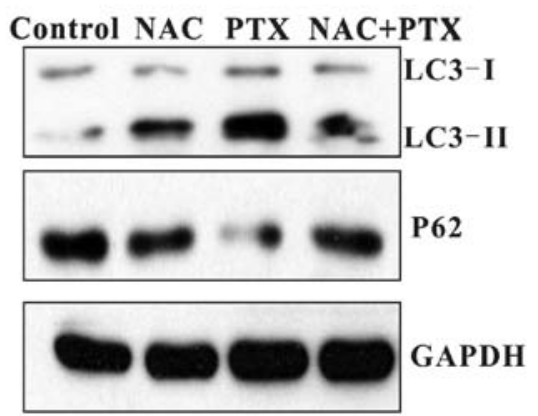

Figure 7. Paclitaxel induces ROS-mediated autophagy in HEC-1A and JEC cells. After pre-treatment with NAC (1 mM) or vehicle for $1 \mathrm{~h}$, HEC-1A and JEC cells were treated with $20 \mu \mathrm{g} / \mathrm{ml}$ paclitaxel or vehicle for $24 \mathrm{~h}$. LC3 conversion and P62 was determined by western blot analysis. 
induced cell death, thereby confirming a protective role of autophagy induced by paclitaxel in certain types of endometrial carcinoma. Based on these findings, it can be hypothesized that autophagy is activated in response to paclitaxel-induced damage to various cellular structures, and that this autophagic process has the unintended result of maintaining the survival of cancer cells and paclitaxel resistance. Previous studies have shown that paclitaxel can induce cytoprotective autophagy in multiple cancer cell lines (8-10), suggesting that the cytoprotective effect of autophagy induced by paclitaxel is not cell-type specific, and may be associated with paclitaxel resistance.

Reactive oxygen species (ROS) are chemically-reactive molecules containing oxygen, which form as a natural byproduct of the normal metabolism of oxygen. When produced in moderate amounts, ROS are thought to operate as signaling molecules in signal transduction pathways regulating cell growth, differentiation, survival, inflammation and the immune response. When produced in excessive amounts, ROS may inflict oxidative damage to vital biological molecules, such as DNA, lipids and proteins, which alters their functionality and causes impairment of cellular integrity. Recently, it has been demonstrated that ROS can induce autophagy (24). Early studies have shown that paclitaxel can induce the generation of ROS in several cancer cell lines, and that this induction of ROS is involved in paclitaxel-induced apoptosis (21-23). Our study also demonstrated that paclitaxel induced ROS generation in a time- and dose-dependent manner. Intriguingly, we found that when ROS levels were decreased using NAC, a general ROS scavenger, autophagy was blocked, indicating that ROS generation mediates autophagy upon paclitaxel treatment.

The generation of ROS by anticancer agents has been reported to mediate autophagy in previous studies (25-29). ROS have been shown to induce autophagy by several processes: activating ERK and JNK, inhibiting mTOR signaling by activating AMP-activated protein kinase (AMPK), and promoting the translocation of oxidative HMGB1 from nucleus to cytoplasm. In addition, arsenic trioxide, a chemical known for inducing autophagic cell death by induction of oxidation stress in several cancer cell lines, was found to stimulate autophagy through upregulating BNIP3, which displaces Bcl-2/Bcl-XL from its complex with Beclin $1(30,31)$. Our study found that Beclin 1 expression increased with autophagy, indicating Beclin 1 complex may be involved in the regulation of paclitaxel induced autophagy in endometrial carcinoma cells. It remains to be further validated whether paclitaxel-induced ROS production promotes autophagy by the activation of multiple pro-autophagic signal pathways or by liberating Beclin 1.

As mounting reports have provided data suggesting that autophagy can facilitate the survival of cancer cells in response to anticancer treatment, autophagy has been proposed as a potential therapeutic target for anticancer drug resistance in the future. To circumvent cytoprotective autophagy, autophagy inhibitors are employed to overcome anticancer therapy resistance, including pharmacological autophagy inhibitor 3-methyladenine (3-MA), bafilomycin A1 (BafA), CQ and its derivatives, as well as siRNA that target essential modulators of autophagic machinery. Among them, CQ and its derivatives have gained increasing attention due to their favorable pharmacological properties and physiological tolerance as evidenced by their long history of use as anti-malarial agents and in diseases such as rheumatoid arthritis. CQ inhibits lysosomal acidification, which blocks the terminal stage of autophagy and has indeed been shown to potentiate the anticancer effects of various chemotherapeutic drugs both in vitro and in vivo (32-35). Currently, multiple clinical trials using CQ as a sensitizing reagent, in combination with standard cancer therapies, are under evaluation in different tumor types, including lung cancer, glioblastoma multiforme, multiple myeloma, breast cancer, melanoma, colon cancer, prostate cancer, and advanced solid tumors unresponsive to chemotherapy (http:// clinicaltrials.gov). In the present study, co-treatment of $\mathrm{CQ}$ and paclitaxel in paclitaxel-insensitive HEC-1A and JEC cells led to autophagy blockage and increased cell death. This result suggests that CQ can enhance the responsiveness of chemotherapeutic-resistant endometrial carcinoma cells to paclitaxel. Thus, treating cancer cells with CQ and its derivatives may provide an important technique to make endometrial carcinoma responsive to paclitaxel chemotherapy.

In conclusion, the results of this study revealed that paclitaxel can increase autophagic activity in paclitaxel-insensitive endometrial carcinoma cells, and that suppressing autophagy could enhance paclitaxel-induced cell death. Taken together, this suggests that autophagy-inhibitor therapy could be an effective and potent strategy to improve paclitaxel treatment outcomes in the treatment of endometrial carcinoma.

\section{Acknowledgements}

This study was supported by grants from the National Nature Science Foundation of China (30772332).

\section{References}

1. Mazgani M, Le N and Hoskins PJ; British Columbia Cancer Agency: Reuse of carboplatin and paclitaxel in patients with relapsed endometrial cancer - the British Columbia Cancer Agency experience. Gynecol Oncol 111: 474-477, 2008.

2. Pectasides D, Xiros N, Papaxoinis G, Pectasides E, Sykiotis C, Koumarianou A, Psyrri A, Gaglia A, Kassanos D, Gouveris P, et al: Carboplatin and paclitaxel in advanced or metastatic endometrial cancer. Gynecol Oncol 109: 250-254, 2008.

3. Sorbe B, Andersson H, Boman K, Rosenberg P and Kalling M: Treatment of primary advanced and recurrent endometrial carcinoma with a combination of carboplatin and paclitaxellong-term follow-up. Int J Gynecol Cancer 18: 803-808, 2008.

4. Vandenput I, Vergote I, Leunen K, Berteloot P, Neven P and Amant F: Leuven dose-dense paclitaxel/carboplatin regimen in patients with primary advanced or recurrent endometrial carcinoma. Int J Gynecol Cancer 19: 1147-1151, 2009.

5. Lefranc F, Facchini V and Kiss R: Proautophagic drugs: A novel means to combat apoptosis-resistant cancers, with a special emphasis on glioblastomas. Oncologist 12: 1395-1403, 2007.

6. Mathew R, Karantza-Wadsworth V and White E: Role of autophagy in cancer. Nat Rev Cancer 7: 961-967, 2007.

7. Morselli E, Galluzzi L, Kepp O, Vicencio JM, Criollo A, Maiuri MC and Kroemer G: Anti- and pro-tumor functions of autophagy. Biochim Biophys Acta 1793: 1524-1532, 2009.

8. Zhang Q, Si S, Schoen S, Chen J, Jin XB and Wu G: Suppression of autophagy enhances preferential toxicity of paclitaxel to folliculindeficient renal cancer cells. J Exp Clin Cancer Res 32: 99, 2013.

9. Kim HJ, Lee SG, Kim YJ, Park JE, Lee KY, Yoo YH and Kim JM: Cytoprotective role of autophagy during paclitaxelinduced apoptosis in Saos-2 osteosarcoma cells. Int J Oncol 42: 1985-1992, 2013

10. Xi G, Hu X, Wu B, Jiang H, Young CY, Pang Y and Yuan H: Autophagy inhibition promotes paclitaxel-induced apoptosis in cancer cells. Cancer Lett 307: 141-148, 2011. 
11. Ajabnoor GM, Crook T and Coley HM: Paclitaxel resistance is associated with switch from apoptotic to autophagic cell death in MCF-7 breast cancer cells. Cell Death Dis 3: e260, 2012.

12. Eum KH and Lee M: Crosstalk between autophagy and apoptosis in the regulation of paclitaxel-induced cell death in v-Ha-rastransformed fibroblasts. Mol Cell Biochem 348: 61-68, 2011.

13. Veldhoen RA, Banman SL, Hemmerling DR, Odsen R, Simmen T, Simmonds AJ, Underhill DA and Goping IS: The chemotherapeutic agent paclitaxel inhibits autophagy through two distinct mechanisms that regulate apoptosis. Oncogene 32: 736-746, 2013.

14. Mizushima N and Yoshimori T: How to interpret LC3 immunoblotting. Autophagy 3: 542-545, 2007.

15. Klionsky DJ, Abdalla FC, Abeliovich H, Abraham RT, AcevedoArozena A, Adeli K, Agholme L, Agnello M, Agostinis P, Aguirre-Ghiso JA, et al: Guidelines for the use and interpretation of assays for monitoring autophagy. Autophagy 8: 445-544, 2012.

16. Pankiv S, Clausen TH, Lamark T, Brech A, Bruun JA, Outzen H, Overvatn A, Bjorkoy $\mathrm{G}$ and Johansen T: p62/SQSTM1 binds directly to Atg8/LC3 to facilitate degradation of ubiquitinated protein aggregates by autophagy. J Biol Chem 282: 24131-24145, 2007.

17. Ichimura $\mathrm{Y}$ and Komatsu M: Selective degradation of $\mathrm{p} 62$ by autophagy. Semin Immunopathol 32: 431-436, 2010.

18. Luiken JJ, Aerts JM and Meijer AJ: The role of the intralysosomal $\mathrm{pH}$ in the control of autophagic proteolytic flux in rat hepatocytes. Eur J Biochem 235: 564-573, 1996.

19. Amaravadi RK, Yu D, Lum JJ, Bui T, Christophorou MA, Evan GI, Thomas-Tikhonenko A and Thompson CB: Autophagy inhibition enhances therapy-induced apoptosis in a Myc-induced model of lymphoma. J Clin Invest 117: 326-336, 2007.

20. Maclean KH, Dorsey FC, Cleveland JL and Kastan MB Targeting lysosomal degradation induces p53-dependent cell death and prevents cancer in mouse models of lymphomagenesis. J Clin Invest 118: 79-88, 2008

21. Alexandre J, Batteux F, Nicco C, Chéreau C, Laurent A, Guillevin L, Weill B and Goldwasser F: Accumulation of hydrogen peroxide is an early and crucial step for paclitaxelinduced cancer cell death both in vitro and in vivo. Int J Cancer 119: 41-48, 2006.

22. Ramanathan B, Jan KY, Chen CH, Hour TC, Yu HJ and Pu YS: Resistance to paclitaxel is proportional to cellular total antioxidant capacity. Cancer Res 65: 8455-8460, 2005.

23. Lyle PA, Mitsopoulos P and Suntres ZE: N-acetyl cysteine modulates the cytotoxic effects of Paclitaxel. Chemotherapy 57: 298-304, 2011

24. Dewaele M, Maes H and Agostinis P: ROS-mediated mechanisms of autophagy stimulation and their relevance in cancer therapy. Autophagy 6: 838-854, 2010.
25. Miki H, Uehara N, Kimura A, Sasaki T, Yuri T, Yoshizawa K and Tsubura A: Resveratrol induces apoptosis via ROS-triggered autophagy in human colon cancer cells. Int J Oncol 40: 1020-1028, 2012.

26. Kim JY, Cho TJ, Woo BH, Choi KU, Lee CH, Ryu MH and Park HR: Curcumin-induced autophagy contributes to the decreased survival of oral cancer cells. Arch Oral Biol 57: 1018-1025, 2012.

27. Yang L, Yang M, Zhang H, Wang Z, Yu Y, Xie M, Zhao M, Liu L and Cao L: S100A8-targeting siRNA enhances arsenic trioxideinduced myeloid leukemia cell death by down-regulating autophagy. Int J Mol Med 29: 65-72, 2012.

28. Lin CJ, Lee CC, Shih YL, Lin TY, Wang SH, Lin YF and Shih CM: Resveratrol enhances the therapeutic effect of temozolomide against malignant glioma in vitro and in vivo by inhibiting autophagy. Free Radic Biol Med 52: 377-391, 2012.

29. Cheng P, Ni Z, Dai X, Wang B, Ding W, Rae Smith A, Xu L, Wu D, He F and Lian J: The novel BH-3 mimetic apogossypolone induces Beclin-1- and ROS-mediated autophagy in human hepatocellular carcinoma [corrected] cells. Cell Death Dis 4: e489, 2013.

30. Kanzawa T, Zhang L, Xiao L, Germano IM, Kondo Y and Kondo S: Arsenic trioxide induces autophagic cell death in malignant glioma cells by upregulation of mitochondrial cell death protein BNIP3. Oncogene 24: 980-991, 2005.

31. Chinnadurai G, Vijayalingam S and Gibson SB: BNIP3 subfamily BH3-only proteins: Mitochondrial stress sensors in normal and pathological functions. Oncogene 27 (Suppl 1): S114-S127, 2008.

32. Bellodi C, Lidonnici MR, Hamilton A, Helgason GV, Soliera AR, Ronchetti M, Galavotti S, Young KW, Selmi T, Yacobi R, et al: Targeting autophagy potentiates tyrosine kinase inhibitorinduced cell death in Philadelphia chromosome-positive cells, including primary CML stem cells. J Clin Invest 119: 1109-1123, 2009.

33. Ni Z, Wang B, Dai X, Ding W, Yang T, Li X, Lewin S, Xu L, Lian J and He F: HCC cells with high levels of Bcl-2 are resistant to ABT-737 via activation of the ROS-JNK-autophagy pathway. Free Radic Biol Med 70: 194-203, 2014.

34. Sun K, Xie X, Liu Y, Han Z, Zhao X, Cai N, Zhang S, Song J and Wei L: Autophagy lessens ischemic liver injury by reducing oxidative damage. Cell Biosci 3: 26, 2013.

35. Guo XL, Li D, Hu F, Song JR, Zhang SS, Deng WJ, Sun K, Zhao QD, Xie XQ, Song YJ, et al: Targeting autophagy potentiates chemotherapy-induced apoptosis and proliferation inhibition in hepatocarcinoma cells. Cancer Lett 320: 171-179, 2012. 